Chirurg 2022 $93: 256$

https://doi.org/10.1007/s00104-022-01609-8

Angenommen: 3. Februar 2022

Online publiziert: 23. Februar 2022

(c) The Author(s), under exclusive licence to Springer Medizin Verlag GmbH, ein Teil von Springer Nature 2022

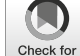

\section{Nachruf auf Prof. Dr. med. Volker Schumpelick}

1944-2022

J. R. Siewert

TUM Emeritus of Excellence, c/o Klinikum rechts der Isar, München, Deutschland
Univ.-Prof.em. Dr. Drs. h.c. J. Rüdiger Siewert em. Direktor der Chirurgischen Klinik und Poliklinik derTUM

em. Ärztlicher Direktor Klinikum rechts der IsarderTUM

em. Ltd. Ärztlicher Direktor des Universitätsklinikums Heidelberg

em. Ltd. Ärztlicher Direktor des UniversitätsklinikumsFreiburg

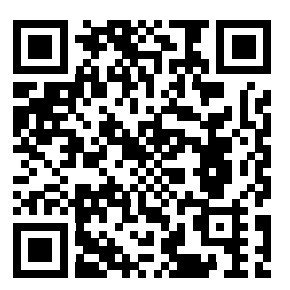

QR-Code scannen \& Beitrag online lesen
In den Augen seiner Kollegen und Weggefährten war Volker Schumpelick - obwohl 1944 in Jena geboren - ein überzeugter und überzeugender Hanseat. Dies ist wohl die Konsequenz seiner medizinischen Ausbildung in Hamburg (1970 medizinisches Staatsexamen, Promotion auch dort 1971). Im selben Jahr trat er seine Ausbildung als Chirurg am Universitätsklinikum in Hamburg Eppendorf unter der Leitung von Hans-Wilhelm Schreiber an. Er habilitierte sich 1978 dort und verblieb bis 1985 an der Hamburger Klinik. Im Jahre 1985 folgte er dann einem Ruf nach Aachen und verblieb dort bis zu seiner Emeritierung im Jahre 2010.

Im Jahre 1983 war sein damaliger Chef H.W. Schreiber Präsident der Deutschen Gesellschaft für Chirurgie. Für die Planung des Kongresses galt es Themen festzulegen. Schreiber entschied unter anderem auch das Thema "Hernien-Chirurgie“ auf die Tagesordnung zu setzen. Er forderte seinen Schüler Schumpelick auf, dieses Thema vorzubereiten. Bis zu diesem Zeitpunkt war das Thema "Hernien-Chirurgie“ nur von geringem Interesse. Schumpelick griff das Thema auf und führte es in die Neuzeit. Er zeigte auf, wie interessant diese Chirurgie sein konnte, wenn man die Fortschritte der Chirurgie auch in diesem Bereich zur Anwendung brachte. Schumpelick wurde dieses Thema nie mehr los und wurde dafür mit Auszeichnungen und hohen Ämtern bedacht.

Insofern war es für Volker Schumpelick eine willkommene Erweiterung seines Spektrums als Schriftleiter der Zeitschrift DerChirurg tätig zu werden. Hier konnte er zeigen, dass er die gesamte Breite der Chirurgie beherrschte und dies durch die Auswahl ganz unterschiedlicher jeweils aktueller Themen unter Beweis stellen konnte. So profitierte nicht nur er, sondern auch die Zeitschrift sehr von dieser Kooperation.

Volker Schumpelick erfuhr in den nächsten Jahren vielerlei Ehrungen und Auszeichnungen und darf mit Recht als einer der renommiertesten und erfolgreichsten deutschen Lehrstuhlinhaber für Chirurgie bezeichnet werden.

Nach seiner Emeritierung in Aachen zog es ihn wieder zurück nach Hamburg in seine alte Heimat. Leider konnte er seinen Ruhestand nicht in vollen Zügen genießen, sondern wurde von einer schweren Erkrankung heimgesucht.

Er ist nun von uns gegangen und möge erlöst von somatischen Problemen seine ewige Ruhe finden. Wir, seine Kollegen und Weggefährten, werden ihm ein ehrendes Angedenken bewahren. Volker Schumpelick war einer der ganz Großen seines Faches.

Univ.-Prof. J.R. Siewert

Februar 2022

\section{Korrespondenzadresse}

Univ.-Prof.em. Dr. Drs. h.c. J. R. Siewert

TUM Emeritus of Excellence, c/o Klinikum rechts der Isar

Ismaninger Straße 22, 81675 München, Deutschland

jr.siewert@tum.de 